\title{
Time-domain signal processing via four-wave mixing in nonlinear delay lines
}

\author{
T. R. O'Meara \\ Amnon Yariv* \\ Hughes Research Laboratories \\ 3011 Malibu Canyon Road \\ Malibu, California 90265
}

\begin{abstract}
The use of nonlinear optical techniques for the temporal processing of information encoded as envelopes on electromagnetic fields is discussed. Applications using these all-optical interactions in the areas of signal processing, radar, and communication systems are considered and compared with existing approaches.
\end{abstract}

Keywords: optical phase conjugation; time-domain nonlinear filters; time reversal in nonlinear delay lines; temporal convolution and correlation; space-to-time encoding; nonlinear matched filters.

Optical Engineering 21(2), 237-242 (March/April 1982).

\section{CONTENTS}

I. Introduction

II. Signal processing systems

A. Collinear geometry and its applications

B. Orthogonal pumping geometry: temporal multiplication, correlation, and convolution applications

C. Orthogonal pumping geometry: envelope time reversal applications

D. Orthogonal pumping geometry with space-to-time encoding

III. Conclusions

IV. Acknowledgment

V. References

\section{INTRODUCTION}

Most of the initial interest in the field of nonlinear conjugation has involved its use as a means of the spatial information processing of electromagnetic fields (such as adaptive optical or real-time holographic processors). ${ }^{1-3}$ There exists another major area of potential practical interest: that of temporal-domain signal processing. In this paper we will demonstrate new techniques for employing nonlinear optical interactions in various signal processing operations on time-domain signals. Among the functions which can be achieved are time-delay control, logic gating, temporal convolution and correlation, envelope reversal, and space-to-time modulation/demodulation. Among the many possible applications of these functions are data processing, pulse encoding/compression (in optical and/or microwave radars), and high bandwidth filtering for communication systems.

*Permanent address: California Institute of Technology, Pasadena, California 91!25.

Invited Paper PC-108 received Oct. 5, 1981; accepted for publication Oct. 14, 1981; received by Managing Editor Oct. 29, 1981.

1982 Society of Photo-Optical Instrumentation Engineers.
The basic geometries involve the interaction of several temporally encoded optical fields within a suitable nonlinear medium. The desired output waveform will be dependent upon the input fields (sequencing and envelopes), directions, phases, amplitudes, and polarizations, as well as the size and shape of the nonlinear medium. All of these applications employ a nonlinear medium for the coupling of the fields. One mechanism that couples the input fields, called four-wave mixing (FWM), can be viewed as a realtime holographic exposure and readout process. Specifically, given a medium that possesses a third-order susceptibility, spatial patterns are "written" into the medium (by the interference of the input waveforms) in the form of spatially varying index gratings.* Since the temporal length of the signals which may be processed using the techniques is proportional to the length of the medium, it is advantageous to configure the nonlinear medium as long, thin slabs or fibers. We shall call such structures nonlinear delay lines. Wide-band nonlinear media are assumed such that these grating patterns may be written in the nonlinear delay lines and erased very rapidly (at over $1 \mathrm{GHz}$ rates), although this is not essential for all applications.

The temporal information being processed or generated is assumed encoded as complex envelopes on one or more optical carriers. A typical $\ell-\mathrm{TH}$ input field is of the form

$\left.E_{p}(z, t)=1 / 2\left\{A_{\rho}\left(t-\frac{z}{v}\right) \exp \left[i \omega_{o} t-k_{p} z\right)\right]+c . c.\right\}$,

wherein the information to be processed is contained in the complex envelope function $A_{\rho}(t-z / v)$. These waveforms can be realized

*We note that these processes are not necessarily limited to nonresonant FWM, nor to spatial grating mechanisms; in general, there are many classes of nonlinear optical in teractions, materials, and physical mechanisms (e.g., multiphoton transitions, magnetic coherences, etc.) that may be employed. 
(for example) via an optical modulator operating on a laser beam. Further, we assume $A_{\rho}(t-z / v)$ to be slowly varying in both the temporal and spatial senses; that is, the spectral width occupied by $A_{\rho}(t)$ is small compared to the carrier frequency $\omega_{0}$. Additionally, we assume in the following discussions that the nonlinear delay line is nondispersive and instantaneously responsive.

Although some of the functions to be demonstrated can be achieved with other than four-wave mixer geometries, there are three important advantages to the four-wave systems: (1) they are extremely versatile; (2) they require only a single wavelength source; and (3) the conjugation action implicit in the four-wave mixer can compensate for path error problems. More specifically, many of the present applications require coherent arithmetic processing (i.e., sum and difference mixing) of the optical waves scattered from different portions of an optical delay line; in typical systems* of this type, path length errors in propagating from these scattering elements must be held to less than $\lambda / 10$ for the summations to be sufficiently precise for systems applications. This constraint can be circumvented by the four-wave mixing systems since path length induced phasing errors (in the component summations) may be eliminated by the conjugation action of the four-wave mixer.

The all-optical nature of these interactions may result in devices possessing increased bandwidth, lower cost and weight, as well as reduced complexity when compared to their electronic counterparts. Finally, we note that the concepts discussed here can be generally extended to other portions of the electromagnetic spectrum by the proper choice of nonlinear media, guided structures, sources, etc.

In this paper we discuss some of the basic signal processing systems together with a simple physical description of the operating principles. In addition some of the implementation advantages and problem areas will be considered. We will find that both timevariant and time-invariant filters can be synthesized by the use of these nonlinear interactions. A detailed mathematical analysis which more precisely quantifies the functional output of these various systems will be the topic of a future publication. ${ }^{6}$ In this analysis we have assumed a weak scattering system which can be approximated by a single differential equation, as contrasted to the usual pair of differential equations describing probe and signal propagation. This contrasts to the time-domain analysis of Fisher ${ }^{7}$ (in this issue of Opt. Eng. 21(2) 1982) which includes both differential equations. Both treatments ignore pump depletion effects.

\section{SIGNAL PROCESSING SYSTEMS}

For all the signal processing systems to be discussed, we employ a degenerate four-wave nonlinear optical mixing process. As in most four-wave systems, there are three input optical waves incident upon a nonlinear medium giving rise to the desired output field. In what follows, we discuss several different device geometries (beam directions, temporal encoding of the beam directions, and nonlinear medium configuration), along with potential device realizations.

\section{A. Collinear geometry and its applications}

Probably the simplest of such systems is illustrated in Fig. 1. It consists of collinear input fields $\left(E_{1,2,4}\right)$ incident into a slab of material possessing a nonlinear refractive index $\chi^{(3)}$. The signals $E_{1}$ and $E_{2}$ are short pulses which are counterpropagating and cross-polarized, with $\mathrm{E}_{4}$ being a short pulse and polarized parallel to $\mathrm{E}_{2}$. As pulse $\mathrm{E}_{2}$ propagates down the nonlinear delay line, it beats with $\mathrm{E}_{4}$, forming a moving (interference) intensity pattern which modulates the nonlinear refractive index of the medium to form an index grating whose envelope replicates the envelope of the moving pulse $E_{2}$. More accurately, this index pattern is a parallel-plane grating structure with fringe patterns whose fine structure remains fixed in space but with an envelope which translates with the velocity of light in the nonlinear medium as illustrated in Fig. 1(b). Returning to Fig.

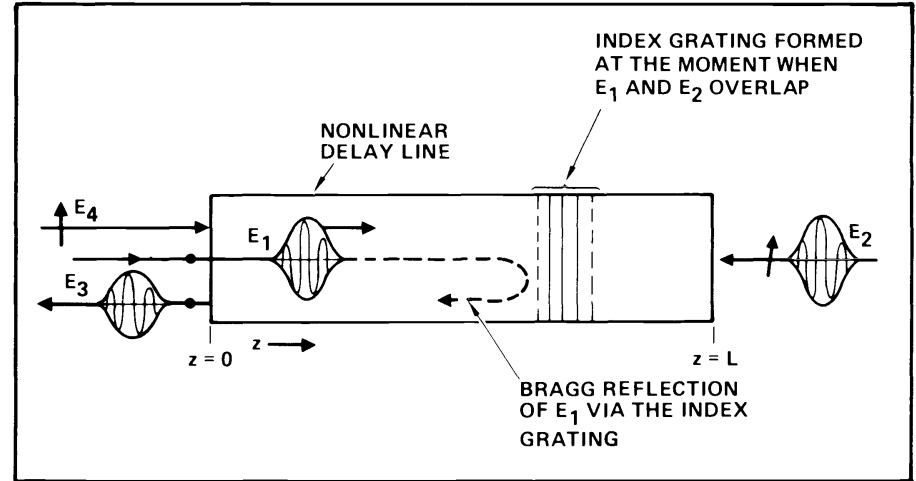

Fig. 1(a). Time-delay control of one optical pulse $\left(E_{3}\right)$ by another $\left(E_{2}\right)$ using a nonlinear delay line with collinear four-wave mixing. The inputs $E_{4}(c w)$ and $E_{2}$ (pulse) are copolarized while the signal pulse to be controlled is orthogonally polarized. The same geometry can be employed as a pulse-shaping network.

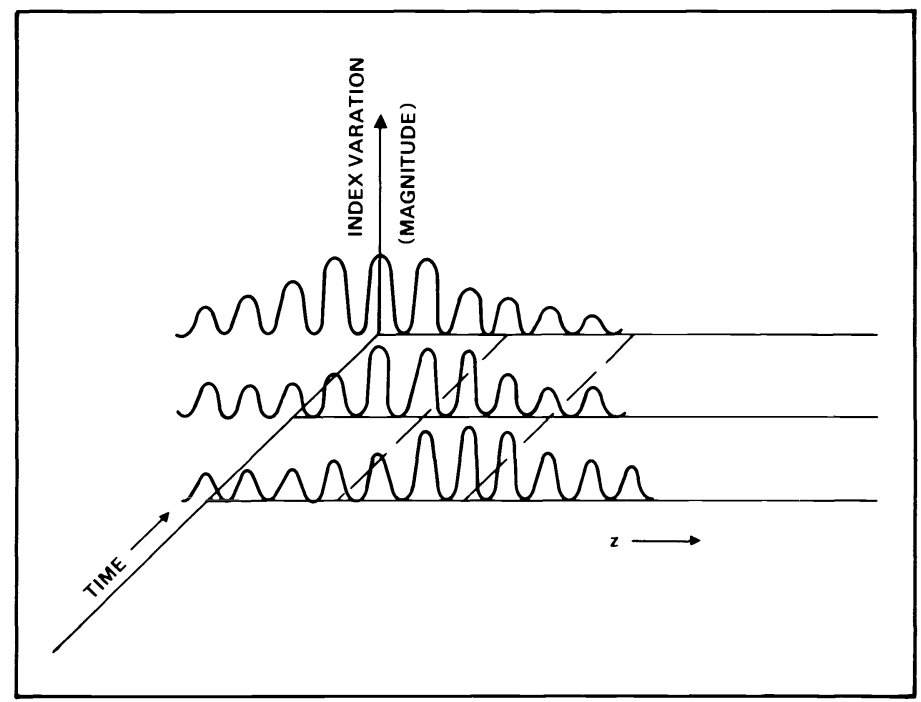

Fig. 1(b). Index grating evolution with time. Note that while the entire envelope of the grating translates to the left (with the velocity of light in the nonlinear medium), the relative position of the in. dividual fringes remains fixed in space.

1(a), a short-duration cross-polarized (pump) pulse $E_{1}$ enters the nonlinear delay line from the opposite side (i.e., relative to the field $E_{2}$ ) and scatters off of an essentially localized grating, giving rise to the output field $E_{3}$. The strength of the backscattered pulse $E_{3}$ can be related to the product of the input fields $E_{4}, E_{2}$ and $E_{1}$ and the nonlinear susceptibility $\chi^{(3)}$ of the nonlinear medium. In particular, it can be shown ${ }^{6}$ that the output field envelope at the input plane $\mathrm{z}=0$ is given by

$A_{3}(0, t)=-i \alpha A_{2}(t) \int_{0}^{L} A_{1}\left(t-\frac{2 z}{v}\right) A_{4}^{*}\left(t-\frac{2 z}{v}\right) d z$,

where

$\alpha=\frac{2 \pi \omega}{\mathrm{cn}} \chi^{(3)}$,

and the fields themselves are of the form given by Eq. (1). Note that this backscatter only occurs at a region centered on that particular position in the delay line wherein the peaks of the $E_{1}$ and $E_{4}$ intersect. If $E_{2}$ is delayed in its entrance time, this crossover position shifts further to the right, and an $E_{1}$ pulse must propagate further 
down the line before it encounters the backscattering diffraction grating pattern formed by $E_{4}$ and $E_{2}$; this delays the exit of $E_{3}$ from the nonlinear line at $z=0$. This action is confirmed by Eq. (2) since a time delay in the leading multiplier $\mathbf{A}_{2}(t)$ generates a corresponding delay in $\mathrm{A}_{3}(0, \mathrm{t})$.

One possible application of this scheme is to an electronically programmable optical delay line. For example, a trigger delay on the laser which generates $E_{2}$ can be used to introduce a relative lead or delay in an existing pulse $\mathrm{E}_{1}$ (whose original timing may not be accessible to direct control).

Other applications are worth considering. If one employs short pulses for all three input fields, then an output pulse $E_{3}$ only occurs if $E_{1}, E_{2}$, and $E_{4}$ exist and overlap. One may use this property to construct logic functions such as "and" or "or," etc. It can be shown that the shape of the $A_{3}$ output envelope pulse can be modified by changing the shape and timing of the envelopes $A_{1}$ and $A_{4}$ (assuming $E_{3}$ to be the input), as well as by selecting a spatially varying nonlinear medium. Thus pulse shaping is another possible application.

\section{B. Orthogonal pumping geometry: temporal multiplication, correlation, and convolution applications}

In this geometry, as illustrated in Fig. 2(a), two of the inputs, $E_{1}$ and $E_{2}$, counterpropagate down the nonlinear delay line. The complex envelopes of these waves represent two signals which are to be multiplied, convolved, or correlated. The third input field $\mathrm{E}_{4}$ is normal to the propagation directions of $E_{1}$ and $E_{2}$; that is, it enters through the side of the delay line. The $\mathrm{E}_{4}$ field is a probe signal which is used to "read out" the product or the convolution/correlation integral, giving rise to the output wave $E_{3}$. This output wave is in the form of a conjugate field scattered off of the grating patterns formed by the beating between $E_{1}$ or $E_{2}$ and the probe field $\mathrm{E}_{4}$

4 . If the probe field $E_{4}$ is a very short pulse plane wave which uniformly illuminates the delay line at the moment of partial or complete overlap of $E_{1}$ and $E_{2}$ within the delay line, then $E_{4}$ beats with $E_{1}\left(E_{2}\right)$ to form an extended grating at $45^{\circ}$ ) which is spatially modulated in the $\mathrm{z}$ direction according to the magnitude of the envelope of $E_{1}\left(E_{2}\right)$. The other field $E_{2}\left(E_{1}\right)$ scatters off of this grating with a strength proportional to the product of its envelope strength $A_{2}(z)$ and the strength of the local fringe pattern, which is in turn proportional to $A_{1}(z)$. Thus the scattered field envelope varies as

$A_{3}(z) \sim A_{1}(z) A_{2}\left(z-z^{\prime}\right)$

where $z^{\prime}$ is a position slip which depends upon the relative timing of the pulses as they enter the delay line. Clearly, if we could collect the entire field $E_{3}(z)$ and integrate it over $z$, we would achieve a signal which had the basic form of a convolution/correlation integral:

$$
C\left(z^{\prime}\right)=\int_{z=0}^{z=L} A_{1}(z) A_{2}\left(z-z^{\prime}\right) d z .
$$

In particular, if we illuminate with a cw probe signal $\mathrm{E}_{4}$ (rather than a pulse), the relative slip between $A_{1}$ and $A_{2}, z^{\prime}$, progresses with time as the waves $E_{1}$ and $E_{2}$ pass each other. More precisely for the case of cw probe illumination, Eq. (5) becomes ${ }^{6}$

$\mathrm{A}_{3}(\mathrm{t}, 0)=\beta \boldsymbol{\Gamma}_{-\infty}^{\infty} \mathrm{A}_{1}(\mathrm{z}-\mathrm{vt}) \mathrm{A}_{2}(\mathrm{z}+\mathrm{vt}) \mathrm{dz}$.

We see that Eq. (6) is of the form of a temporal correlation function.

The integration over $\mathrm{z}$ is easily achieved with a lens $\mathrm{L}_{1}$ as illustrated in Fig. 2(b). Note that although $\mathrm{L}_{1}$ may need to be large, it need not be high quality, since the conjugation action implicit in

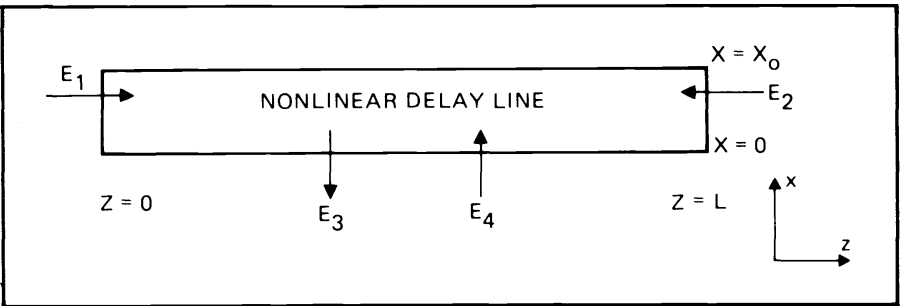

Fig. 2(a). The four-wave mixer as a multiplier. The output $E_{3}(z, t)$ is given by the product of $E_{1}(z, t)$, and $E_{2}(z, t) ; E_{4}$ is assumed constant in space and time.

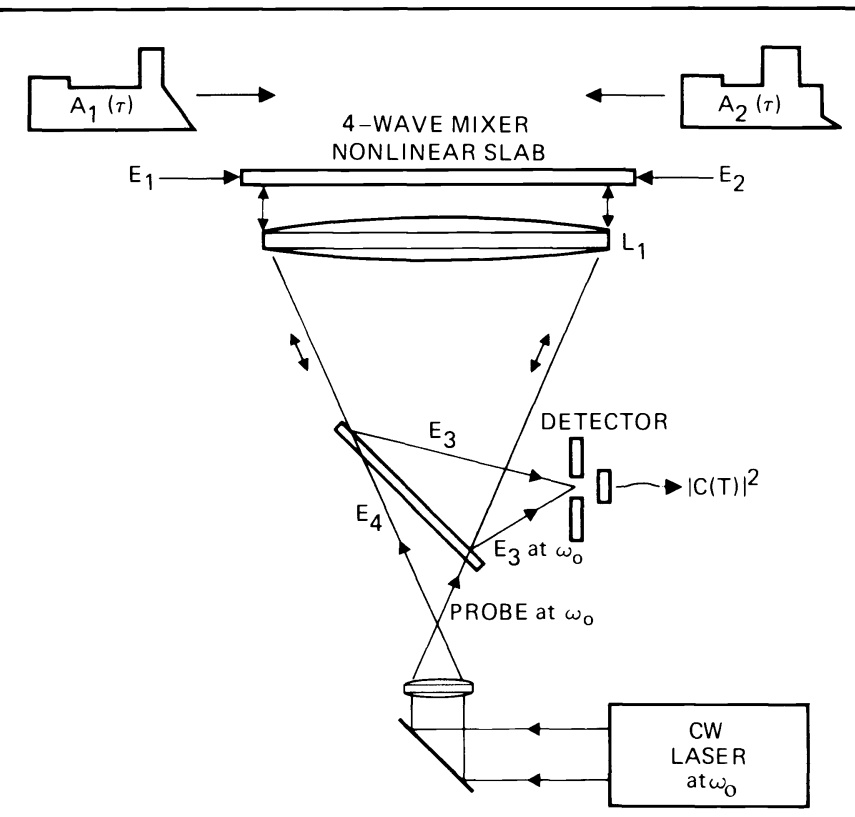

Fig. 2(b). The four-wave mixer as a time-domain correlator. The modulation envelopes $A_{1}(\tau)$ and $A_{2}(\tau)$ are cross-correlated in the nonlinear slab as they pass each other, and the detector output $\left.C(\tau)\right|^{2}$ gives the correlation function as a function of time.

the nonlinear delay line eliminates the lens distortions. Similarly there is no requirement that the nonlinear delay line be particularly flat.*

It should be emphasized that, since we are counting on accurate summations over various time slices of the modulated carriers, the wave trains (and operations on them) must remain fully coherent for Eq. (6) to be valid. The conjugate nature of the signal does not relax this requirement. The fields $\mathrm{E}_{1}$ and $\mathrm{E}_{2}$ would, therefore, typically be produced by two modulators acting on a single laser source.

Also note that the multiplication in the four-wave mixer product is a field multiplication. Thus, assuming that a coherent or a heterodyne detection is employed, the optical modulator should modulate the input field linearly with respect to voltage or current. If a conventional direct detector is employed (as illustrated), then modulators should be employed which generate intensities proportional to current or voltage. This approach causes a loss of sign in the correlation output. If negative correlation values are of interest, one must substitute a heterodyne detector for the direct detector; the sign of the correlation value is then encoded on the phase of the intermediate frequency output.

As a further practical matter, one must closely coordinate the entry of the $E_{1}$ and $E_{2}$ fields into the opposite ends of the nonlinear

*Without conjugation, the path lengths involved in the coherent integration of Eq. (6) would typically have to be controlled to $\lambda / 10$ or better. 
delay lines since, with long pulses, too large a timing error will produce optical vignetting (clipping of the integration range).

Long input pulses, $\mathrm{E}_{1}$ and $\mathrm{E}_{2}$, can be accommodated by substituting a nonlinear fiber for the nonlinear slab and coiling it within the probe lens illumination area.

\section{Orthogonal pumping geometry: envelope time reversal applications}

The basic system of Fig. 2(a) can also be employed for envelope time reversal as illustrated in Fig. 3. In this case, pump pulses $E_{3}$ and $\mathrm{E}_{4}$ are very short and coherent, and are extended in the $\mathrm{z}$ direction to pump the entire nonlinear volume. They are also timed to overlap in the nonlinear delay line. We assume that the probe field $E_{1}$ is also a coherent optical wave train with an envelope which possesses a distinctive nonsymmetrical structure, as illustrated in Fig. 3(a). We desire to time-reverse the envelope of the field $E_{1}$. This envelope is assumed to be long compared to the pump pulse duration, yet sufficiently short to "fit" within the nonlinear delay line. We further arrange the timing such that, at the moment that the entire envelope of the probe pulse $E_{1}$ is contained within the nonlinear delay line, the pump fields are pulsed to overlap in the nonlinear delay line. At this moment, an extended diffraction grating is formed by the beat between $E_{1}$ and $E_{3}\left(E_{4}\right)$ in the nonlinear medium, whose strength (which varies with $\mathrm{z}$ ) replicates the envelope of the probe pulse, as illustrated by Fig. 3(a). This grating scatters a portion of the pump pulse $E_{4}\left(E_{3}\right)$ backwards in the direction of $E_{2}$ (Fig. 3(b)). If the backscattering grating decays (as a consequence of the pump pulses passing out of the nonlinear line) before the probe field $E_{1}$ has propagated over a distance equal in length to its smallest scale size, then the backscattered pulse essentially replicates the probe pulse in shape. However, not only is the envelope replicated in shape, it is reversed in time as illustrated in Fig. 3(b). In a manner of speaking, the last part of the wave train to enter the nonlinear roundhouse must back out first.

The maximum resolution of the system may potentially be limited by the response time of the nonlinear medium as well as by the temporal duration of the pump pulses. The analysis of this case, as given by D. A. B. Miller, ${ }^{8}$ results in an output field whose amplitude is given by

$A_{3}\left(-t-\frac{z}{v}\right)=\frac{-i 8 \pi \omega}{n^{2} c}\left(\frac{2 \pi \chi^{(3)}}{n}\right) F A_{4}^{*}\left[-\left(t-2 t_{p}\right)-\frac{z}{v}\right]$,

where $t_{p}$ is the temporal extent of the input pulse, $F$ is the temporal correlation of the pump pulses, and $v=c / N$. The parameter $A_{4}(t)$ is the time-dependent, complex amplitude of the input probe waveform.

\section{Orthogonal pumping geometry with space-to-time encoding}

The objective in this class of systems is to synthesize an arbitrary filter response function in the time-domain sense. That is, a short probe pulse-an "impulse-like" input-is to produce a prescribed output response function* in the form of an envelope on the backscattered (conjugate) signal.

As illustrated in Fig. 4, the structural approach is to employ spatial phase and/or amplitude encodings on one or both pump signals $E_{1}$ and $E_{2}$, which are translated into corresponding temporal modulations on the signal $\mathrm{E}_{3}$ envelope via the cross-coupling in the nonlinear delay line. More specifically, two cw counterpropagating pump waves $E_{1}(z)$ and $E_{2}(z)$ having specified $z$-dependent complex amplitudes are incident along the $x$ direction on the nonlinear delay time. Typically, only one pump would be so encoded; the second pump would simply replicate the spatial pattern on the first via a mirror reflection. For the particular example of Fig. 4, one pump $E_{2}$ is a plane wave and the other $\left(E_{1}\right)$ is encoded

*In linear time-domain network synthesis, this output is called the impulse response function of the filter.

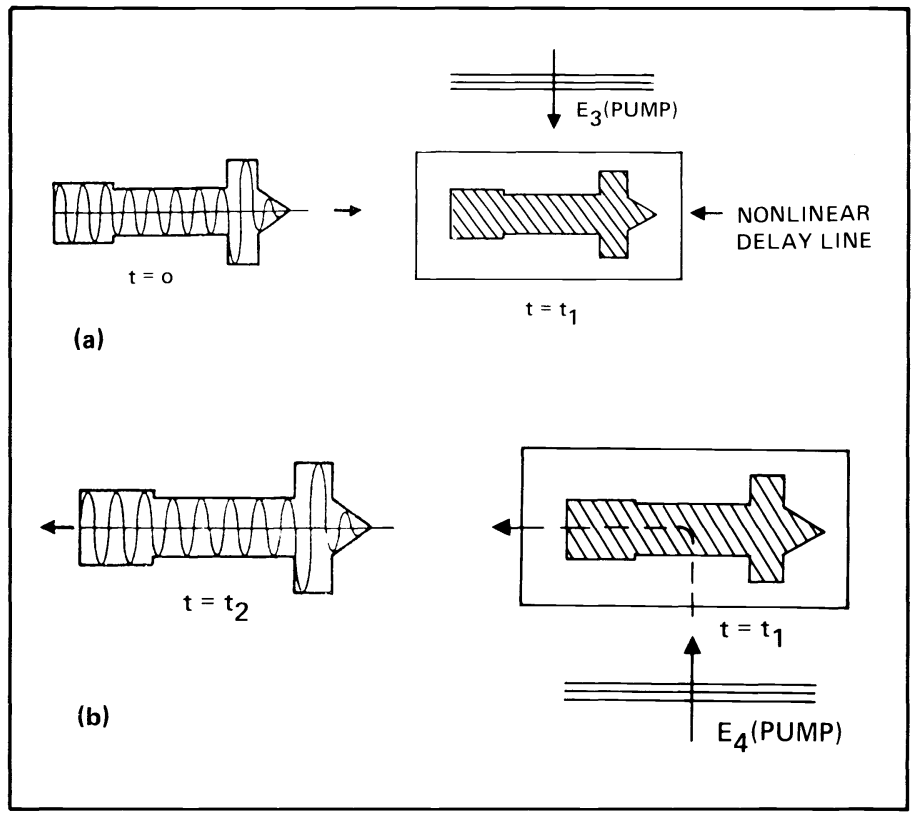

Fig. 3. Wave-train time reversal with a nonlinear delay line. (a) Holographic recording process: the wave-train amplitude modulates the strength of the holographic fringe pattern which fills the nonlinear delay line. (b) Holographic playback process: the wave-train amplitude is retained in the playback process, wherein the opposing pump reflects off of the hologram.

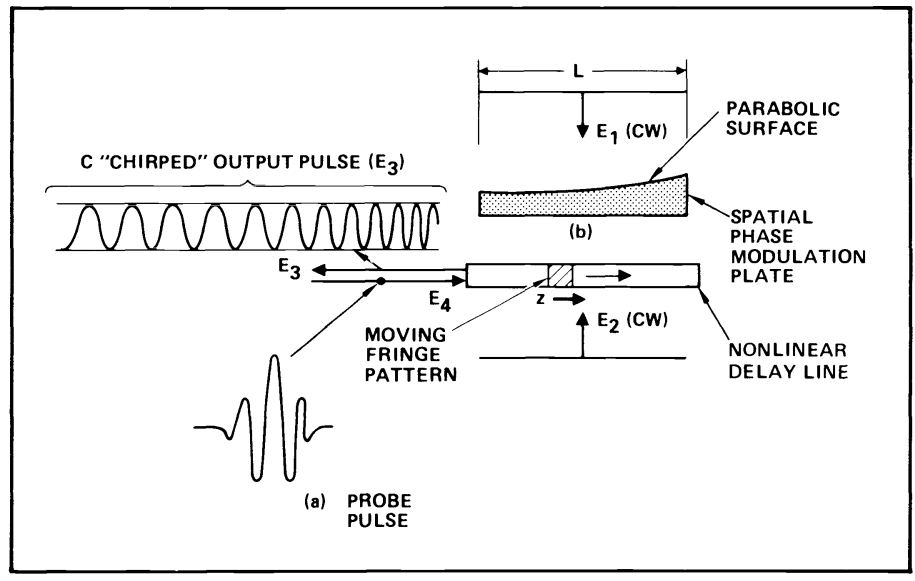

Fig. 4. Spatial-to-temporal encoding. In this example, a (parabolic) spatial phase encoding on pump $E_{1}$ scatters off of an index grating formed by the beat between probe $E_{4}$ and pump $E_{2}$ to become $a$ temporal encoding - a parabolic phase or chirp-on the output envelope $E_{3}$.

with a z-dependent parabolic phase distribution. A short probe pulse $\mathrm{E}_{4}$ (Fig. 4(a)) travels down the nonlinear delay line and generates a moving fringe pattern by beating against one of the pumps $\left(E_{2}\right)$. The other pump $E_{1}$ scatters off of this grating as it progresses down the nonlinear delay line and forms the backscattered signal* $\mathrm{E}_{3}$. This backscattered signal carries the phase and amplitude of the $E_{1}$ pump at every position $z$ where it is backscattered. Further, since the backscattered signal arriving from point $\mathrm{z}$ is delayed in time by

$\mathrm{t}=\frac{2 \mathrm{z}}{\mathrm{v}}$,

where $\mathrm{v}$ is the velocity of light in the nonlinear line, this positional

*A similar operation occurs from the beat between $E_{4}$ and $E_{1}$. 
phase information is encoded as a temporal modulation as indicated by Fig. 4(c). The total encoded pulse length thus corresponds to the maximum delay, which is

$\mathrm{T}_{\mathrm{p}}=\frac{2 \mathrm{~L}}{\mathrm{v}}$,

where $\mathrm{L}$ is the length of the nonlinear delay line (or the pump width if this is smaller).

For the "chirp",9 example of Fig. 4, the pump phase variation in $\mathrm{z}$ is represented by its complex amplitude

$A_{1}(z)=a_{1} \exp \left[i \phi_{p}\left(\frac{z}{L}\right)^{2}\right]$,

and the envelope of the output signal is accordingly proportional to

$A_{3}(0, t) \sim P(t) \exp \left[i \phi_{p}\left(\frac{t}{2 T_{p}}\right)^{2}\right]$,

where

$P(t)=\left\{\begin{array}{l}1 ; 0<t<T_{p} \\ 0 ; \text { otherwise }\end{array}\right\}$,

which is a "chirped" FM waveform as illustrated in Fig. 4(c). Alternative spatial and temporal modulations are readily produced. The exact form of $\mathrm{A}_{3}(0, \mathrm{t})$ for a general pump modulation is derived in Ref. 6.

As a time domain "'network," this system is linear with respect to its input signal $E_{4}$. That is, under the operating assumptions stated in the introduction, the system obeys superposition with respect to the input-output signals. Further, in contrast to some of the systems previously considered, a delay in the input pulse which enters the nonlinear line yields an output which is correspondingly delayed in time and which has the same response in all other respects. In other words, it is time invariant. As a consequence, all of the operations of linear, time-independent filter theory are equally applicable to this system. For example, the response to an arbitrary driving signal $\mathrm{E}_{4}$ is given by the convolution of this signal with the impulse response of the filter.

Given this fact, one may borrow from the existing literature of linear filter theory to generate prescribed filters. For example, "matched filter" pairs are readily synthesized where the matched filter ${ }^{10}$ has an impulse response which is the time reverse of the impulse response of the original filter. Thus, the "matched filter" for the chirp filter of Fig. 5 is essentially the same system as for the original filter with the encoding phase distribution on pump $E_{1}$ reversed in space. In fact, any spatial encoding and its (z direction) reversed encoding constitutes a matched filter pair. Furthermore, any such filter can constitute its own matched filter, if the probe pulse enters into the system from the reverse end* of the nonlinear delay line to that which was employed to generate the original filter response.

For chirp filters and other suitably chosen modulation functions, the combined process of first processing by the original encoding filter followed by a processing via the matched filter approximately reproduces the original impulse. 9 That is, the original "stretched" output pulse of length $T_{p}$ is "pulse-compressed" back to a short impulse (with, however, some spurious, low-level side-lobe structure, which extends over a time $2 \mathrm{~T}_{\mathrm{p}}$ ).

So far, we have assumed that the pump spatial encodings which specify the impulse response of the filter are fixed, such that we have a time-independent response. A more general class of filter

* In contrast, entering a signal into an ordinary dispersive transmission filter from the back end produces exactly the same filter response as from the forward end.

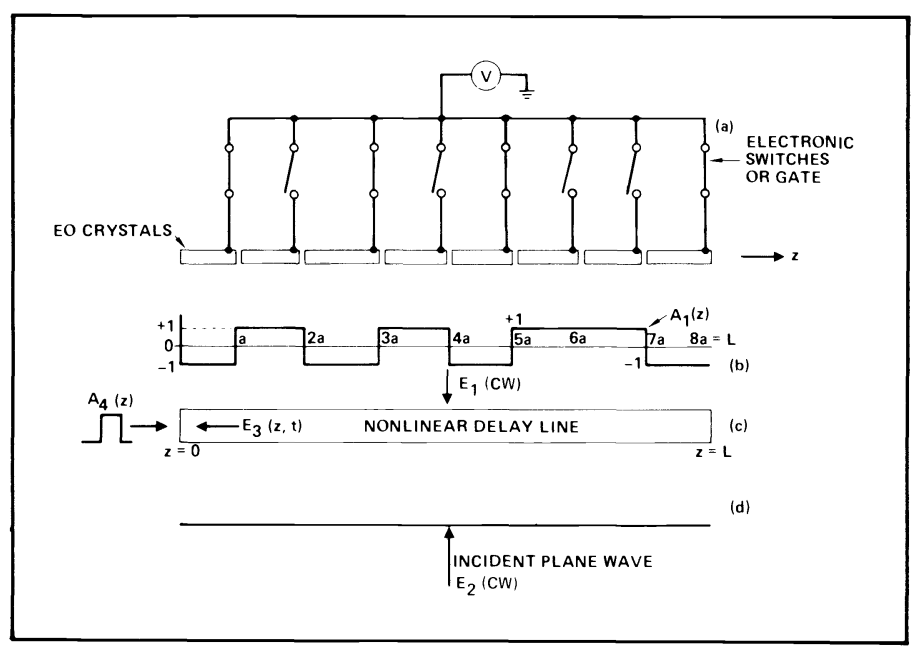

Fig. 5. A programmable nonlinear delay line filter which employs spatial-to-temporal encoding. The application of a spatially depen. dent (digital) voltage sequence (a) to a linear array of electro-optic elements results in a spatially encoded phase front (b) upon prop. agation of a cw plane wave $E_{1}$ through the network. In the example, this wave mixes with a cw plane wave $E_{2}$, as shown by (d), and a pulse field $A_{4}(z)$, all within a nonlinear medium (c). The output field $E_{3}$ is thus temporally encoded; the result is shown in Fig. 6.

may be synthesized by substituting one or more electro-optic (E-O) crystals for the fixed phase plate as illustrated in Fig. 5. Such a system may generate a $\mathrm{z}$ dependent phase shift proportional to applied voltage. The result is a very general filter whose impulse response may be programmed in time. On application is to generate high data rate communication signals, as illustrated in Fig. 5, with the output for this example shown in Fig. 6 . The parallel modulation results in an effective modulation of the output envelope at rates which can exceed, by a large factor, the capability of the individual electro-optic crystals. If the typical length of the crystal is, say, $3 \mathrm{~mm}$, then the individual pulses in Fig. 5 are $\sim 30$ picoseconds. The rise time of the voltage applied to each E-O crystal need be no shorter than $\sim \mathrm{L} / \mathrm{v}$, which, in this example, could correspond to a nanosecond.

There is an important bonus which results from the fact that the backscattered signal $E_{3}$ is conjugated. For example, consider the use of pulse compression for encoding and decoding. In such systems, to achieve high quality performance, the dispersed components of $E_{3}$ must be brought back into a state of coherence by the matched filter. That is, the phase shift on the leading part of the pulse must be exactly controlled relative to that of the lagging part if these components are to be brought into phase synchronism. In a nonconjugation system of this class, the propagation delays to the end of the delay line and back impact this synchronism. Thus, in such a system, if the delay line undergoes temperature change, such that it expands by $\lambda / 4$, the relative phasing between the lending and lagging components is destroyed. In contrast, for the four-wave conjugation systems, as proposed in this paper, the extra path expansion induced phase shift is exactly compensated by the process of conjugation and retransmission back over this same expanded path.

Some practical problems are worth mentioning. If the nonlinear conjugation efficiency is too high, and if the gratings have a duration which is long compared to the probe transit time, then one can have secondary scatterings ${ }^{11}$ off of successive cells in the conjugated output encoded or decoded signal, which could degrade the performance of the system. Thus the nonlinear gratings should be weak.

Even with weak gratings, the probe will be attenuated as it propagates down the line, both as a consequence of scattering and absorption losses in the nonlinear media. One way to overcome this problem is to progressively increase the strength of the pumps as 


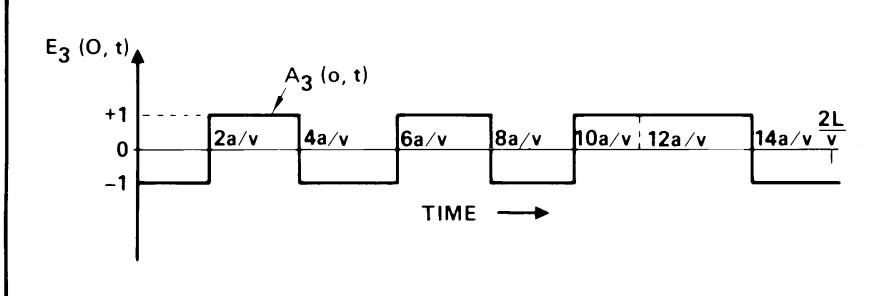

Fig. 6. All illustrative output wave train generated by a program. mable nonlinear delay line, resulting from the encoding sequence shown in Fig. 5.

the feed paths progress from the input to the output end of the nonlinear transmission line.

\section{CONCLUSIONS}

We have defined a number of novel time-domain signal processing applications which exploit the multiplicative properties inherent in the four-wave mixing process. Further, we have demonstrated how spatial information encoded on an optical wavefront can be transformed into equivalent coherent temporal encoding on a second optical carrier. All of these operations have the potential of being realized at very large bandwidths. The four-wave mixers offer a unique capability for coherent processing with delay line filters since path errors in the coherent summation process can be effectively eliminated by the conjugation action inherent in the fourwave mixing process.

\section{ACKNOWLEDGMENT}

The authors wish to gratefully acknowledge the helpful assistance of D. M. Pepper, whose contributions extend far beyond the usual bounds of editorial comment.

\section{REFERENCES}

1. J. O. White and A. Yariv, "Real-Time Adaptive Optics and Spatial Information Processing via Four-Wave Mixing," this issue of Opt. Eng. 21(2) 1982.

2. T. O'Meara, "Compensation of Laser System Distortions with Nonlinear Conjugators," this issue of Opt. Eng. 21(2) 1982

3. D. Hon, "Compensation of Laser System Distortions with Nonlinear Conjugators," this issue of Opt. Eng. 21(2) 1982.

4. IEEE Transactions on MTT, Vol. MTT-17, No. 11, Special Issue on Microwave Acoustics (Nov. 1969).

5. H. E. Kallmann, Proc. IRE, 28, 302 (1940).

6. T. O'Meara and A. Yariv, "Analysis of Time Domain Signal Processing via Four-Wave Mixing,"' to be published.

7. B. R. Suydam, and Robert A. Fisher, "Transient Response of KerrLike Phase Conjugators: a Review,' this issue of Opt. Eng. 21(2) 1982.

8. D. A. B. Miller, Opt. Lett. 5, 300 (1980).

9. C. E. Cook and M. Bernfeld, Radar Signals-an Introduction to Theory and Application, Chap. 6, Academic Press, New York (1967).

10. Op. Cit, pp. 5-17.

11. The analysis of such "strong" coupling between the probe and conjugate waves requires a simultaneous solution of a pair of differential equations describing their respective evolutions; see Ref. 7 above for more details. 